\title{
Hopelessness, Depression, and Early Markers of Endothelial Dysfunction in U.S. Adults
}

\author{
D. Phuong Do, PhD, Jennifer Beam Dowd, PhD, Nalini Ranjit, PhD, James S. House, PhD, And \\ George A. Kaplan, PhD
}

\begin{abstract}
Objective: To examine whether the psychological traits of hopelessness and depressive symptoms are related to endothelial dysfunction. Methods: Data are derived from a subsample of 434 respondents in the 2001 to 2003 Chicago Community Adult Health Study, a population-based survey designed to study the impact of psychological attributes, neighborhood environment, and socioeconomic circumstances on adults aged $\geq 18$ years. Circulating biomarkers of endothelial dysfunction, including e-selectin, p-selectin, and soluble intercellular adhesion molecule-1 (s-ICAM1) were obtained from serum samples. Hopelessness was measured by responses to two questions, and depressive symptoms were measured by an 11-item version of the Center for Epidemiological Studies Depression scale. Multivariate regression models tested whether continuous levels of the biomarkers (natural log transformed) were associated with levels of hopelessness and depressive symptoms separately and concurrently. Results: In age- and sex-adjusted models, hopelessness showed significant positive linear associations with s-ICAM1. In contrast, there was no significant linear association between hopelessness and e-selectin and p-selectin. Adjustment for clinical risk factors, including systolic pressure, chronic health conditions, smoking, and body mass index, did not substantively alter these associations. Results from similar models for depressive symptoms did not reveal any association with the three biomarkers of endothelial dysfunction. The associations between hopelessness and e-selectin and s-ICAM1 were robust to the inclusion of adjustments for depressive symptoms. Conclusions: Negative psychosocial traits may influence cardiovascular outcomes partially through their impact on the early stages of atherosclerosis, and specific psychosocial traits, such as hopelessness, may play a more direct role in this process than overall depressive symptoms. Key words: hopelessness, depression, endothelial dysfunction, biomarkers, cardiovascular disease.
\end{abstract}

CCAHS = Chicago Community Adult Health Study; s-ICAM1 = soluble intercellular adhesion molecule-1; CVD = cardiovascular disease.

\section{INTRODUCTION}

$P$ revious work has shown that psychosocial factors, including hopelessness and depression, predict hypertension incidence, atherosclerotic progression, ischemic heart disease, and acute myocardial infarction (1-5). However, little is known about whether these psychosocial factors also predict subclinical measures of endothelial dysfunction, an early precursor of atherosclerosis. Understanding the pathophysiological mechanisms linking psychosocial factors to cardiovascular disease is an important step in continued cardiovascular disease (CVD) prevention efforts.

Endothelial dysfunction reflects a vascular phenotype prone to atherogenesis, serving as an important predictor of cardiovascular events (6). Dysfunction of the endothelium typically leads to immunological alterations, including activation, adhesion, and

From the Department of Health Services Policy and Management (D.P.D.), Arnold School of Public Health, University of South Carolina, Columbia, South Carolina; School of Public Health, Hunter College (J.B.D.), City University of New York (CUNY); CUNY Institute for Demographic Research (J.B.D.), New York, New York; University of Texas School of Public Health (N.R.), Austin Regional Campus, Austin, Texas; Michael \& Susan Dell Center for Advancement of Healthy Living (N.R.), Austin, Texas; Department of Sociology (J.S.H.), Ford School of Public Policy (J.S.H.), Institute for Social Research (J.S.H.), Center for Social Epidemiology and Population Health (G.A.K.), and Department of Epidemiology (G.A.K.), School of Public Health, University of Michigan, Ann Arbor, Michigan.

Address correspondence and reprint requests to D. Phuong Do, $\mathrm{PhD}$, Department of Health Services Policy and Management, University of South Carolina, 800 Sumter St., Columbia, SC 29208. E-mail: dphuongdo@sc.edu

Received for publication June 26, 2009, revision received March 11, 2010.

This research was supported, in part, by Grant P50HD38986 and R01HD050467 from the National Institute of Child Health and Human Development and Grant P0117943 from the Kellogg Health Scholars Program (D.P.D.). Additional support was provided by The Robert Wood Johnson Health and Society Scholars Program (J.B.D.).

DOI: 10.1097/PSY.0b013e3181e2cca5 aggregation of platelets to areas of damage. Attachment of monocytes and lymphocytes to endothelial cells is mediated by cellular adhesion molecules, comprised of the selectin family (e-selectin, p-selectin, and l-selectin) and the immunoglobulin group, including intercellular adhesion molecule-1 $(7,8)$. Selectins are involved in the first step of leukocyte adhesion at sites of inflammation or injury, whereas the intercellular adhesion molecule mediates tight attachment of leukocytes and platelets to the endothelium (9). Levels of soluble adhesion molecules reflect this process of inflammation of the vessel wall and have been found to predict cardiovascular events in healthy populations (10-12).

A sense of hopelessness is characterized by a sense of futility and negative expectations about the future and one's personal goals. Hopelessness seems to lead to more negative evaluations of new situations and less effective coping strategies (10). Although hopelessness is often a major symptom of depression, it may play a distinct and larger role in the development of CVD risk (2). Negative emotions, such as hopelessness, may influence the progression of atherosclerosis through chronic stimulation of the sympathetic nervous system and the hypothalamic-pituitaryadrenocortical axis (11-14), which can injure the intima and vascular endothelial cells (15-17). Thus far, little work has directly tested associations of hopelessness and inflammatory markers, with one study (18) finding no relationship in a subsample of the Whitehall II cohort. In another study (19), plasma fibrinogen was found to be a moderate-strength mediator of the association between hopelessness and progression of carotid atherosclerosis in Finnish men. Nonetheless, numerous studies (20-22) have pointed to a relationship between psychosocial stress, negative emotions, and increases in systemic inflammatory markers, including C-reactive protein, interleukin-6, and tumor necrosis factor (TNF)- $\alpha$. Because adhesion molecules are thought to express on the endothelial membrane partly in response to several inflammatory cytokines (23), we hypothesized that hopelessness will be associated with circulating levels of these adhesion molecules. 
Depression is characterized by a depressed mood, loss of interest or pleasure, feelings of guilt or low-self worth, disturbed sleep or appetite, and low energy. Several pathways have been proposed to explain observed links between depression and CVD. Depression may be associated with unhealthy life-styles, such as poor diet and lack of exercise, and nonadherence to treatment regimens. Depression has been associated with higher levels of C-reactive protein, interleukin-6, and TNF- $\alpha$, and increased monocyte expression of proinflammatory cytokines and chemokines, and thus may foster inflammation that contributes to CVD (24), although some studies $(25,26)$ have found no association of depression with inflammatory markers. With regard to endothelial function, depression has been found to be associated with impaired flow-mediated dilatation of the brachial artery (4). Depression may act as a chronic stressor that contributes to endothelial dysfunction through abnormalities in cellular adhesion, migration, and proliferation (4). Depression has previously been linked to higher levels of intercellular adhesion molecule-1, e-selectin, and monocyte chemoattractant protein- 1 in a clinical case-controlled study (27). Given the existing evidence on depression and inflammatory pathways, we expected that depression will be associated with markers of endothelial dysfunction in our sample.

To the best of our knowledge, no previous study has examined the effect of hopelessness on markers of subclinical endothelial dysfunction. One previous study (27) examined the link between depression and e-selectin in a clinical sample, but to our knowledge, the association between depression and a broader set of endothelial markers in a population-based sample has not been characterized. The objective of this study was to examine whether hopelessness and depression are related to endothelial dysfunction, as measured by serum values of three markers of endothelial function: e-selectin and p-selectin, and soluble intercellular adhesion molecule-1 (s-ICAM1).

\section{METHODS}

\section{Sample Population}

Data for this study are derived from the first wave of the Chicago Community Adult Health Study (CCAHS), a population-based survey designed to study the impact of psychological attributes, neighborhood environment, and socioeconomic circumstances on adults aged $\geq 18$ years, conducted from 2001 to 2003. Sociodemographic and psychosocial data were collected on the full CCAHS sample $(n=3105)$ during the interview portion of the study. Full details of the CCAHS have been reported elsewhere (28). All subjects gave informed consent for both the interviews and serum collection, and these studies were approved by the University of Michigan School of Public Health Institutional Review Board. Blood samples were drawn by certified phlebotomists for a random subsample of 661 respondents from 80 neighborhoods, frozen at least $-20^{\circ} \mathrm{C}$ by Quest Laboratories (Chicago, Illinois), and shipped to the Central Ligand Assay Satellite Services Laboratory (Ann Arbor, Michigan), where they were stored at $-70^{\circ} \mathrm{C}$ until the time of analysis. All three biomarkers were assayed with enzyme-linked immunosorbent assay kits (R\&D systems, Minneapolis, Minnesota). We excluded individuals who reported ever having a heart attack or had missing values on any of the biomarkers or independent variables. The resulting final sample size for analyses consisted of 434 observations.

\section{Measures}

Key Independent Variables: Hopelessness was measured with two items from the Kuopio Ischemic Heart Disease study that captures negative expectations about oneself and the future: 1) "I feel it is impossible for me to reach the goals I would like to strive for." 2) "The future seems hopeless to me and I can't believe that things are changing for the better." These two items have been used in previous studies $(1,2,29)$ that examined the relationship between hopelessness and CVD. For each item, respondents answered on a scale of 1 to 4 whether they agreed strongly with the statement, agreed somewhat, disagreed somewhat, or disagreed strongly. Each response was then reverse coded and summed. We used the sum score to reflect a continuous measure of hopelessness. In addition, in order to capture possible nonlinear effects, we examined a categorical classification of hopelessness level specified by the following delineation. The summed scores (range, $2-8$ ) were divided into three categories (low hopelessness, score $=$ 2; medium hopelessness, $3 \geq$ score $\leq 5$; high hopelessness, $6 \geq$ score $\leq 8$. A score of 2 reflected consistent strong disagreement with each statement, scores from 3 to 5 indicated a more mixed response, and scores from 6 to 8 reflected general agreement with the statements. Although not identical due to differences in scale, we base our grouping to reflect the degree of disagreement with the hopelessness measures that have been employed in previous studies $(1,2)$.

Depressive symptoms were measured by a shortened version of the Center for Epidemiological Studies-Depression Scale (CES-D), which was developed for use in the Iowa cohort of the Established Populations for Epidemiologic Study of the Elderly survey. This shortened version of the scale employs 11 of the 20 items used in the long form of the CES-D; the 11 question items are included in the Appendix (30,31). For each question, respondents were asked to answer on a scale of 1 to 4 the frequency in which they experienced certain feelings during the past week. The frequency ranged from 1) never; 2) hardly ever; 3) some of the time; and 4) most of the time. Similar to our strategy with the hopelessness measure, we used both a continuous score and a categorical specification to examine possible threshold effects. The continuous score reflected the sum of the scores from the 11 items. Two questions that had asked about positive feelings (i.e., happiness, enjoyment of life) were first reverse coded before being summed. For the categorical specification, we first transformed the score distribution to reflect the distribution of the 20 -item scale in a nationally represented sample, using the procedure recommended by Kohout and colleagues (30). We standardized male and female scores separately through a $\mathrm{T}$ score transformation, which shifted the mean and standard deviation (SD) to those from the National Health and Nutrition Examination Survey-I (men: mean, 7.1; SD, 7.2; women: mean, $10.0 ; \mathrm{SD}, 9.1)$. We then created a binary indicator based on the standard cutoff score of 16, reflecting high depressive symptoms (32).

\section{Outcome}

Outcome measures include three markers of endothelial function: pselectin $(\mathrm{ng} / \mathrm{mL})$, e-selectin $(\mathrm{ng} / \mathrm{ml})$, s-ICAM1 $(\mathrm{ng} / \mathrm{mL})$. Due to their skewed distributions, each marker was natural log transformed for all analyses.

\section{Covariates}

We adjusted for a series of individual sociodemographic and health characteristics, including the following: gender; age (continuous); inflammatory disease (binary indicator of whether the respondent has ever been diagnosed with asthma, arthritis, ulcer, or emphysema); CVD (binary indicator of whether the respondent has ever been diagnosed with heart condition, stroke, peripheral artery disease, or diabetes); systolic blood pressure level (continuous); body mass index (BMI) category: normal, $\mathrm{BMI}<25$; overweight, $25 \geq \mathrm{BMI}<30$; obese, $\mathrm{BMI} \geq 30$; and smoking status (never smoked, past smoker, current smoker).

\section{Statistical Analysis}

We estimated a series of multivariable regression models examining the relationship between each marker of endothelial dysfunction and levels of hopelessness and depression, separately and then concurrently. In primary analyses, hopelessness and depressive symptoms were modeled continuously. To capture possible nonlinear effects, as well as to allow meaningful interpretations of differences in outcomes between high and low levels of psychosocial risk, the same models were then repeated with levels of hopelessness and depressive symptoms specified categorically, as described above.

We first estimated a set of models that adjusted for only age and gender (Model 1). Because existing health conditions may lead to hopelessness or depression, we then adjusted for systolic blood pressure level, self-reported 
PSYCHOSOCIAL FACTORS AND ENDOTHELIAL DYSFUNCTION

TABLE 1. Selected Characteristics (Percent or Mean) of Study Participants by Levels of Hopelessness

\begin{tabular}{|c|c|c|c|c|}
\hline Characteristic & $\begin{array}{l}\text { Low Hopelessness, } \\
\qquad(n=171)\end{array}$ & $\begin{array}{l}\text { Medium Hopelessness, } \\
\qquad(n=191)\end{array}$ & $\begin{array}{l}\text { High Hopelessness, } \\
\qquad(n=72)\end{array}$ & $p^{a}$ \\
\hline Gender & & & & .03 \\
\hline Male & 36.85 & 55.26 & 43.27 & \\
\hline Female & 63.15 & 44.74 & 56.73 & \\
\hline Age & $40.72(15.69)$ & $43.90(16.62)$ & $48.15(15.96)$ & .07 \\
\hline \multicolumn{5}{|l|}{ Health conditions } \\
\hline Systolic blood pressure & $120.86(20.13)$ & $123.24(22.28)$ & $127.96(24.27)$ & .44 \\
\hline Cardiovascular condition & 12.78 & 16.29 & 36.56 & .008 \\
\hline Inflammatory condition & 27.62 & 34.02 & 42.45 & .30 \\
\hline \multicolumn{5}{|l|}{ Health behaviors } \\
\hline Body Mass Index & & & & .96 \\
\hline Normal & 31.12 & 29.79 & 31.03 & \\
\hline Overweight & 39.12 & 36.38 & 33.90 & \\
\hline Obese & 29.76 & 33.83 & 35.07 & \\
\hline Smoking & & & & .37 \\
\hline Never smoked & 63.40 & 57.25 & 46.46 & \\
\hline Past smoker & 17.56 & 16.58 & 18.19 & \\
\hline Current smoker & 19.04 & 26.17 & 35.35 & \\
\hline
\end{tabular}

Standard deviation shown in parentheses.

${ }^{a}$ The $p$ values reflect differences across categories as derived from $F$ tests for continuous variables, and $\chi^{2}$ tests for categorical variables.

TABLE 2. Selected Characteristics (Percent or Mean) of Study Participants by Levels of Depressive Symptoms

\begin{tabular}{llll}
\hline Characteristic & $\begin{array}{c}\text { Low } \\
\text { Depressive } \\
\text { Symptoms, } \\
(n=331)\end{array}$ & $\begin{array}{c}\text { High } \\
\text { Depressive } \\
\text { Symptoms, } \\
(n=103)\end{array}$ & $p^{a}$ \\
\hline $\begin{array}{l}\text { Gender } \\
\text { Male }\end{array}$ & 49.98 & 28.14 & .003 \\
Female & 50.02 & 71.86 & \\
Age & $42.73(16.41)$ & $44.86(15.80)$ & .39 \\
Health conditions & & & \\
Systolic blood & $122.71(21.94)$ & $123.58(21.11)$ & .79 \\
$\quad$ pressure & & 30.98 & .003 \\
$\quad \begin{array}{l}\text { Cardiovascular condition } \\
\text { Inflammatory condition }\end{array}$ & 14.44 & 41.96 & .13 \\
Health behaviors & 30.25 & & .97 \\
Body Mass Index & & 30.77 & \\
$\quad$ Normal & 30.46 & 35.75 & \\
$\quad$ Overweight & 37.53 & 33.48 & \\
$\quad$ Obese & 32.00 & 57.13 & \\
Smoking & & 10.10 & \\
$\quad$ Never smoked & 58.67 & 32.77 & \\
$\quad$ Past smoker & 18.87 & & \\
$\quad$ Current smoker & 22.46 & & \\
\hline
\end{tabular}

Standard deviation shown in parentheses.

${ }^{a}$ The $p$ values reflect differences across categories as derived from $F$ tests for continuous variables, and $\chi^{2}$ tests for categorical variables.

inflammatory conditions, and self-reported cardiovascular conditions (Model 2). Finally, because deleterious health behaviors might confound or be a result of hopelessness or depression and contribute to endothelial dysfunction, we included BMI level and smoking status in the models as potential confounders/mediators (Model 3). This sequence of models was first conducted for hopelessness and depressive symptoms separately and then, because hopelessness and depressive symptoms are known to be correlated, we estimated the same set of models with both hopelessness and depression specified concurrently to assess their independent influences.
Further models that included low-density lipoprotein cholesterol level and income were estimated as sensitivity analyses to test the robustness of our results, but they were not included in the primary analyses due to the amount of missing data and subsequent loss of power.

\section{RESULTS}

Simple cross tabulations for hopelessness and depressive symptoms with various sample characteristics were first conducted. Selected sample descriptives by hopelessness levels are presented in Table 1. A higher proportion of individuals indicating either low or high hopelessness was female, and the average age increased with levels of hopelessness. Moreover, a higher proportion of those with high hopelessness reported having an existing health condition (i.e., cardiovascular condition, inflammatory condition), had higher systolic blood pressure, were obese, and currently smoked.

The pattern found for levels of depressive symptoms was similar, with some notable exceptions (Table 2). Similar to hopelessness, a higher proportion of those with high levels of depressive symptoms was female, and the average age was higher among the high depressive symptoms group. A considerably higher proportion of those with high levels of depressive symptoms reported having a cardiovascular or inflammatory condition and currently smoked. However, levels of systolic blood pressure and rates of obesity were similar for the low and high depressive symptoms groups.

Simple correlation analyses revealed that the continuous measures of hopelessness were significantly correlated with e-selectin $(r=.13, p=.001)$ and s-ICAM1 $(r=.15, p=.002)$ but not p-selectin $(r=.00, p=.96)$. In contrast, depressive symptoms were not significantly correlated with any of the biomarkers $(r=$ $.04, p=.44$ with e-selectin; $r=-.01, p=.88$ with p-selectin; $r=.05, p=.28$ with s-ICAM1).

Results from regression models indicated that hopelessness, specified continuously, was significantly associated 
with only one of the three endothelial markers (Table 3 ). The age- and gender-adjusted models suggested a strong positive association with s-ICAM1 in which each additional point of hopelessness was associated with a $3.36 \%$ increase in levels of s-ICAM1. The magnitude of the association was consistently reduced with each set of additional adjustments but remained significant on the addition of controls for health conditions and health behaviors. No significant association was found for e-selectin or p-selectin when hopelessness was measured continuously.

Analyses specifying categorical measures of hopelessness suggested a stepwise relationship between hopelessness and two of the endothelial dysfunction markers. Relative to the lowest level, respondents in the highest level of hopelessness had $>18 \%$ higher levels of e-selectin and s-ICAM1-although the association for e-selectin was only marginally significant. No association was found between hopelessness levels and p-selectin. Adjustments for health conditions (i.e., CVD conditions, noninflammatory conditions, systolic blood pressure) did not alter the associations (Model 2) (Table 3), nor did the inclusion of BMI and smoking status in the final models (Model 3) (Table 3). The association between high hopelessness and e-selectin became statistically significant with the full set of adjustments, and the association between a medium level of hopelessness and e-selectin became margin-

TABLE 3. Regression Model Results of Hopelessness on Biomarkers of Endothelial Dysfunction

\begin{tabular}{|c|c|c|c|}
\hline & $\begin{array}{l}\text { E-Selectin } \\
\% \text { Change }\end{array}$ & $\begin{array}{l}\text { P-Selectin } \\
\% \text { Change }\end{array}$ & $\begin{array}{l}\text { S-ICAM1 } \\
\% \text { Change }\end{array}$ \\
\hline \multicolumn{4}{|l|}{$\begin{array}{c}\text { Continuous hopelessness } \\
\text { measure }\end{array}$} \\
\hline Model 1: Age+gender & 2.93 & 0.65 & $3.36^{a}$ \\
\hline $\begin{array}{l}\text { Model 2: Age }+ \text { gender }+ \\
\text { health conditions }\end{array}$ & 2.49 & 0.44 & $3.10^{a}$ \\
\hline $\begin{array}{c}\text { Model 3: Age }+ \text { gender }+ \\
\text { health conditions }+ \\
\text { health behaviors }\end{array}$ & 2.57 & 0.07 & $2.52^{a}$ \\
\hline \multirow{2}{*}{\multicolumn{4}{|c|}{$\begin{array}{c}\text { Categorical hopelessness } \\
\text { levels } \\
\text { Model 1: Age }\end{array}$}} \\
\hline & & & \\
\hline Low & [ref] & {$[\mathrm{ref}]$} & [ref] \\
\hline Medium & 10.32 & -0.14 & 8.37 \\
\hline High & $18.63^{b}$ & 0.55 & $18.15^{a}$ \\
\hline \multicolumn{4}{|l|}{$\begin{array}{l}\text { Model 2: Age }+ \text { gender }+ \\
\text { health conditions }\end{array}$} \\
\hline Low & [ref] & [ref] & [ref] \\
\hline Medium & $10.59^{b}$ & 0.04 & 7.34 \\
\hline High & $16.08^{b}$ & -0.63 & $17.50^{a}$ \\
\hline \multicolumn{4}{|l|}{$\begin{array}{l}\text { Model 3: Age }+ \text { gender }+ \\
\text { health conditions }+ \\
\text { health behaviors }\end{array}$} \\
\hline Low & [ref] & {$[\mathrm{ref}]$} & [ref] \\
\hline Medium & $10.66^{b}$ & -0.28 & 8.21 \\
\hline High & $17.96^{a}$ & -1.81 & $17.60^{a}$ \\
\hline
\end{tabular}

$n=434 ;$ S-ICAM1, soluble intercellular adhesion molecule-1.

${ }^{a} p<.05$.

${ }^{b} p<.10$.
TABLE 4. Regression Model Results of Depression on Biomarkers of Endothelial Dysfunction

\begin{tabular}{|c|c|c|c|}
\hline & $\begin{array}{l}\text { E-Selectin } \\
\% \text { Change }\end{array}$ & $\begin{array}{l}\text { P-Selectin } \\
\% \text { Change }\end{array}$ & $\begin{array}{l}\text { S-ICAM1 } \\
\% \text { Change }\end{array}$ \\
\hline \multicolumn{4}{|l|}{ Continuous CES-D measure } \\
\hline Model 1: Age+gender & 0.17 & -0.05 & 0.21 \\
\hline $\begin{array}{l}\text { Model 2: Age + gender }+ \\
\text { health conditions }\end{array}$ & 0.08 & -0.08 & 0.03 \\
\hline $\begin{array}{c}\text { Model 3: Age }+ \text { gender }+ \\
\text { health conditions }+ \\
\text { health behaviors }\end{array}$ & 0.08 & -0.21 & -0.18 \\
\hline \multicolumn{4}{|l|}{ Categorical CES-D levels } \\
\hline \multicolumn{4}{|l|}{ Model 1: Age } \\
\hline Low & [ref] & {$[\mathrm{ref}]$} & {$[\mathrm{ref}]$} \\
\hline High & 2.49 & 0.72 & 0.91 \\
\hline \multicolumn{4}{|l|}{$\begin{array}{l}\text { Model 2: Age }+ \text { gender }+ \\
\text { health conditions }\end{array}$} \\
\hline Low & [ref] & {$[\mathrm{ref}]$} & [ref] \\
\hline High & 0.75 & -0.05 & 0.41 \\
\hline \multicolumn{4}{|l|}{$\begin{array}{l}\text { Model 3: Age }+ \text { gender+ } \\
\text { health conditions }+ \\
\text { health behaviors }\end{array}$} \\
\hline Low & [ref] & {$[\mathrm{ref}]$} & {$[$ ref $]$} \\
\hline High & 1.91 & -0.42 & -1.23 \\
\hline
\end{tabular}

$n=434$; S-ICAM1, soluble intercellular adhesion molecule-1; CES-D, Center for Epidemiological Studies Depression scale.

ally statistically significant with the inclusion of the additional set of controls.

The results for age- and sex-adjusted models for depressive symptoms did not support any association with endothelial dysfunction. Both the continuous and categorical specifications of depressive symptoms yielded nominal (i.e., point estimates were close to zero) associations with the three biomarkers and none were significant. The inclusion of additional adjustments did not significantly alter these findings (Table 4).

Regression models with hopelessness and depressive symptoms included together suggested that not only was the association of hopelessness and endothelial dysfunction independent of depressive symptoms, it is strengthened on adjustment. In the continuous models, the magnitude of the point estimates for hopelessness increased for all three biomarkers, compared with comparable models without adjusting for depressive symptoms (Table 5). A single point increase in the hopelessness score was associated with an increase of between $3 \%$ to $4 \%$ for e-selectin and s-ICAM1. However, consistent with models not adjusting for depressive symptoms, hopelessness was found to be significantly associated only with sICAM1. However, in the categorical models, high levels of hopelessness were found to be significantly associated with e-selectin (compared with marginal significance in previous models without controlling for depressive symptoms) across all three model specifications (Table 6). Medium levels of hopelessness were marginally associated with e-selectin. High levels of hopelessness remained significantly associated with s-ICAM1 on the inclusion of depressive symptoms. As before, no association was found between hopelessness and p-selectin 
TABLE 5. Regression Model Results of Continuous Measures of Hopelessness and Depressive Symptoms (Estimated Concurrently) on Biomarkers of Endothelial Dysfunction

\begin{tabular}{|c|c|c|c|}
\hline & $\begin{array}{l}\text { E-Selectin } \\
\% \text { Change }\end{array}$ & $\begin{array}{l}\text { P-Selectin } \\
\% \text { Change }\end{array}$ & $\begin{array}{l}\text { S-ICAM1 } \\
\% \text { Change }\end{array}$ \\
\hline \multicolumn{4}{|c|}{$\begin{array}{c}\text { Continuous hopelessness and } \\
\text { depression measures }\end{array}$} \\
\hline \multicolumn{4}{|c|}{ Model 1: Age+gender } \\
\hline Hopelessness & 3.31 & 0.91 & $3.78^{a}$ \\
\hline CES-D & -0.24 & -0.17 & -0.26 \\
\hline \multicolumn{4}{|c|}{$\begin{array}{l}\text { Model 2: Age }+ \text { gender }+ \\
\text { health conditions }\end{array}$} \\
\hline Hopelessness & 2.90 & 0.69 & $3.75^{a}$ \\
\hline CES-D & -0.27 & -0.17 & -0.43 \\
\hline \multicolumn{4}{|c|}{$\begin{array}{l}\text { Model 3: Age }+ \text { gender }+ \\
\text { health conditions }+ \\
\text { health behaviors }\end{array}$} \\
\hline Hopelessness & 2.97 & 0.45 & $3.40^{a}$ \\
\hline CES-D & -0.27 & -0.26 & -0.59 \\
\hline
\end{tabular}

$n=434$; S-ICAM1, soluble intercellular adhesion molecule-1; CES-D, Center for Epidemiological Studies Depression scale.

TABLE 6. Regression Model Results of Categorical Measures of Hopelessness And Depressive Symptoms (Estimated Concurrently) on Biomarkers of Endothelial Dysfunction

\begin{tabular}{|c|c|c|c|}
\hline & $\begin{array}{l}\text { E-Selectin } \\
\% \text { Change }\end{array}$ & $\begin{array}{l}\text { P-Selectin } \\
\% \text { Change }\end{array}$ & $\begin{array}{l}\text { S-ICAM1 } \\
\% \text { Change }\end{array}$ \\
\hline \multicolumn{4}{|c|}{$\begin{array}{c}\text { Categorical hopelessness } \\
\text { and depressive } \\
\text { symptoms levels }\end{array}$} \\
\hline \multicolumn{4}{|c|}{ Model 1: Age } \\
\hline Low & [ref] & {$[\mathrm{ref}]$} & [ref] \\
\hline Medium & $11.14^{b}$ & -0.24 & $9.45^{b}$ \\
\hline High & $21.40^{a}$ & 0.22 & $21.90^{a}$ \\
\hline \multicolumn{4}{|c|}{ Depressive symptoms } \\
\hline Low & [ref] & [ref] & [ref] \\
\hline High & -4.44 & 0.66 & -5.95 \\
\hline \multicolumn{4}{|c|}{$\begin{array}{l}\text { Model 2: Age }+ \text { gender }+ \\
\text { health conditions } \\
\text { Hopelessness }\end{array}$} \\
\hline Low & [ref] & [ref] & {$[\mathrm{ref}]$} \\
\hline Medium & $11.55^{b}$ & 0.02 & 8.39 \\
\hline High & $19.25^{a}$ & -0.72 & $21.09^{a}$ \\
\hline \multicolumn{4}{|c|}{ Depressive symptoms } \\
\hline Low & [ref] & [ref] & [ref] \\
\hline High & -5.32 & 0.17 & -5.94 \\
\hline \multicolumn{4}{|c|}{$\begin{array}{l}\text { Model 3: Age }+ \text { gender }+ \\
\text { health conditions }+ \\
\text { health behaviors }\end{array}$} \\
\hline \multicolumn{4}{|c|}{ Hopelessness } \\
\hline Low & [ref] & {$[\mathrm{ref}]$} & [ref] \\
\hline Medium & $11.44^{b}$ & -0.31 & 7.33 \\
\hline High & $20.60^{a}$ & -1.91 & $19.18^{a}$ \\
\hline \multicolumn{4}{|c|}{ Depressive symptoms } \\
\hline Low & [ref] & [ref] & [ref] \\
\hline High & -4.45 & 0.20 & -6.87 \\
\hline
\end{tabular}

$n=434 ;$ S-ICAM1, soluble intercellular adhesion molecule-1.

${ }^{a} p<.05$.

${ }^{b} p<.10$. in the categorical models. Depressive symptoms remained unassociated with all three biomarkers in both the continuous and categorical models.

\section{DISCUSSION}

This study identified associations between hopelessness and two of three adhesion molecules thought to be early markers of endothelial dysfunction, a precursor to atherosclerosis. Significant associations of hopelessness with s-ICAM1 were found, net of an extensive list of controls for other risk factors for atherosclerosis, including smoking, obesity, hypertension, and existing chronic conditions in both the continuous and categorical models. A weaker link was found between hopelessness and e-selectin, where high hopelessness was revealed to be significantly associated with e-selectin only after controlling for gender, health conditions, and health behaviors. Hopelessness was not found to be linearly associated with e-selectin. In contrast, we found no evidence to support a link between hopelessness and p-selectin. The differing results across the three biomarkers were not surprising, given the range of correlation strengths; $\mathrm{p}$-selectin and sICAM1 were not correlated $(r=.027, p=.569)$; -selectin and e-selectin had a low correlation $(r=.172, p<.001)$; and e-selectin and s-ICAM1 had the strongest correlation $(r=$ $.314, p<.001)$.

The lack of an association between hopelessness and p-selectin may suggest distinct pathways of influence; in contrast to s-ICAM1 and e-selectin that are activated by inflammatory cytokines, p-selectin has been considered most importantly a marker of platelet activation stimulated by thrombin and adenosine diphosphate $(33,34)$. In addition, biological determinants of p-selectin differ from those of e-selectin. Ponthieux et al. (7) found e-selectin to be positively associated with glucose and TNF- $\alpha$, whereas p-selectin was negatively associated with these markers. Moreover, there are gender differences in p-selectin, but not e-selectin (7). Finally, e-selectin increases with BMI, while p-selectin does not show such an association (7).

We found no evidence that depressive symptoms were associated with any of the three endothelial markers. These results are in contrast to the one existing study that examined the relationship between depression and adhesion molecules. Rajagopalan et al. (27) found depression to be positively associated with s-ICAM and e-selectin. This study was a case-control clinical study of young adult patients with major depressive disorder rather than a population-based study of depressive symptoms, which may account for the different findings. Additional models with a higher CES-D cutoff point of 20, suggestive of higher degree of depression, were estimated as sensitivity analyses; no association with endothelial biomarkers was found in any of these models. Recent work (35) identified a stronger relationship between somatic items (such as poor appetite and fatigue) of the 20-item CES-D scale and future risk of cardiovascular events in women with existing CVD compared with cognitive/affective symptoms. As 
somatic items comprised only two of our 11 CES-D items, this may help explain our negative findings for depression.

Sensitivity analyses were conducted to assess the robustness of our findings for hopelessness. Given cholesterol is a known risk factor for endothelial dysfunction, we tested the robustness of the associations to the inclusion of low-density lipoprotein cholesterol level, with very similar results. Furthermore, because socioeconomic conditions may be correlated with hopelessness, we estimated models that included the respondent's income as a possible confounder or mediator (results not shown); the significant associations remained for s-ICAM1 even after adjustment for this socioeconomic measure. However, the associations for e-selectin were attenuated to nonsignificance, suggesting that income confounded or mediated the relationship between hopelessness and e-selectin. In summary, we found hopelessness to be consistently associated with s-ICAM1, independent of health measures and behaviors, including depressive symptoms. A weaker association was found for e-selectin and only presented when comparing high levels with low levels of hopelessness. No association between hopelessness and p-selectin was found.

The results for hopelessness suggest that negative psychosocial traits may influence cardiovascular outcomes partially through their impact on early stages of atherosclerosis. Our findings are consistent with work (19) that found plasma fibrinogen to be a moderate-strength mediator of the association between hopelessness and progression of carotid atherosclerosis in Finnish men. Fibrinogen is an acute-phase reactant and marker of inflammation that strongly affects platelet aggregation and may also be an indication of injury to vascular endothelial cells (36). Overexpression of the cell adhesion molecules examined in this study may, in part, represent a response to inflammatory stimuli (37). There is very little existing literature on the relationship of hopelessness to inflammatory markers (with one exception) (18); future work should confirm whether inflammation mediates the relationship between hopelessness and endothelial function, or whether other pathways are more salient.

The distinct results for hopelessness and depression suggest that hopelessness may reflect a unique component of depression that may be more relevant to cardiovascular function than measures of depressive symptoms. It is possible that hopelessness reflects a long-term consequence of high levels of depressive symptoms and, thus, reflects a more severe state. The correlation between our measures of hopelessness and depressive symptoms was only .43 $(p<.001)$. The relatively low correlation between these two measures supports the notion that hopelessness and depressive symptoms do not go hand in hand and that hopelessness may be a sufficient but not necessary component of depression. Specifically, hopelessness may be indicative of high depressive symptoms, whereas the reverse may not be true.

The relative strength of the associations found for hopelessness versus depressive symptoms has also been found by several other studies $(1-3,29,38)$ examining CVD. For example, studies have found hopelessness to be associated with incident myocar- dial infarction and cardiovascular mortality (1) and faster progression of carotid atherosclerosis over a 4-year follow-up (3). These associations were not substantially altered after controlling for depressive symptoms. In studies that examined hopelessness and depressive symptoms separately, hopelessness was found to be a stronger predictor of incident fatal and nonfatal ischemic heart disease in both men and women (38).

The puzzle of how and why hopelessness is uniquely associated with CVD, independent of depressive symptoms, is further complicated by consistent findings that traditional risk factors do not mediate the association between hopelessness and CVD (1-3). Our findings were consistent with this pattern, with no confounding or mediating influence of obesity, hypertension, or smoking in our observed relationship between hopelessness and adhesion molecules. Because these risk factors were measured at one point in time and likely measured with error, our models may not reflect the full influence of these factors. Additional risk factors not measured here may be important mechanisms, such as cortisol secretion, inflammatory cytokines, dietary-influenced factors, such as homocysteine, or shared genetic factors (14).

Nonetheless, it is becoming increasingly evident that hopelessness is an important and unique contributor to CVD. We hypothesize that hopelessness, compared with depressive symptoms, reflects a more chronic and permanent attribute that may progressively affect physiological pathways over time. However, our cross-sectional study does not allow us to test this hypothesis. Future studies, using longitudinal data that have information on the dynamics (e.g., onset, duration) of respondents' psychosocial states, may help to reveal why hopelessness is such a strong predictor of cardiovascular-related health outcomes. Moreover, longitudinal analyses can begin to examine to what extent income mediates or confounds the relationship with e-selectin. Although a strength of our study is the use of a population rather than clinical sample, our sample is limited to a discrete geographic area that may be not generalizable to the U.S. population. In addition, the sample sizes of respondents with high hopelessness and depression are relatively small, which may be problematic for detecting a given effect size.

Despite this study's weaknesses, our findings both support and contribute to previous studies examining the relationship between psychosocial factors and CVD. Although previous papers have identified associations between hopelessness and later stages of atherosclerosis and its progression as measured by intima-media thickening (3), this is the first study to examine the association of hopelessness with the cellular adhesion molecules s-ICAM1, e-selectin, and p-selectin. Our results suggest that hopelessness may be a risk factor in early identification of those with higher risk for cardiovascular-related health problems. Associations were strongest for s-ICAM1, weaker for e-selectin, and absent for p-selectin. Future studies should work to replicate these findings, investigate why the association between hopelessness and endothelial markers vary across different cellular adhesion molecules, and identify additional mechanisms linking hopelessness to endothelial dysfunction. 


\section{APPENDIX. CES-D 11 Question Items}

After each statement, please indicate how often you felt that way DURING THE PAST WEEK. Again, the best answer is usually the one that comes to your mind first, so do not spend too much time on any one statement.

Possible Responses: 1) Never; 2) Hardly ever; 3) Some of the time; 4) Most of the time.

\begin{tabular}{|c|c|}
\hline CESD 1 & $\begin{array}{l}\text { I felt depressed. Would you say you felt this way never, } \\
\text { hardly ever, some of the time, or most of the time } \\
\text { during the past week? }\end{array}$ \\
\hline CESD 2 & $\begin{array}{l}\text { I felt that everything I did was an effort. (Would you } \\
\text { say you felt this way never, hardly ever, some of the } \\
\text { time, or most of the time during the past week?) }\end{array}$ \\
\hline CESD 3 & My sleep was restless. \\
\hline CESD 4 & $\begin{array}{l}\text { I was happy. (Would you say you felt this way never, } \\
\text { hardly ever, some of the time, most of the time } \\
\text { during the past week?) }\end{array}$ \\
\hline CESD 5 & I felt lonely. \\
\hline CESD 6 & People were unfriendly. \\
\hline CESD 7 & I enjoyed life. \\
\hline CESD 8 & $\begin{array}{l}\text { I did not feel like eating. My appetite was poor. } \\
\text { (Would you say you felt this way never, hardly ever, } \\
\text { some of the time, or most of the time during the } \\
\text { past week?) }\end{array}$ \\
\hline CESD 9 & I felt sad. \\
\hline CESD 10 & I felt that people disliked me. \\
\hline CESD 11 & I could not get "going." \\
\hline
\end{tabular}

CES-D, Center for Epidemiological Studies Depression scale.

\section{REFERENCES}

1. Everson SA, Goldberg DE, Kaplan GA, Cohen RD, Pukkala E, Tuomilehto J, Salonen JT. Hopelessness and risk of mortality and incidence of myocardial infarction and cancer. Psychosom Med 1996;58:113-21.

2. Everson SA, Kaplan GA, Goldberg DE, Salonen JT. Hypertension incidence is predicted by high levels of hopelessness in Finnish men. Hypertension 2000;35:561-7.

3. Everson SA, Kaplan GA, Goldberg DE, Salonen R, Salonen JT. Hopelessness and 4-year progression of carotid atherosclerosis: the Kuopio ischemic heart disease risk factor study. Arterioscler Thromb Vasc Biol 1997; 17:1490-5.

4. Strike PC, Steptoe A. Psychosocial factors in the development of coronary artery disease. Prog Cardiovasc Dis 2004;46:337-47.

5. Gallo LaKAM. Do negative emotions mediate the association between socioeconomic status and health? Ann N Y Acad Sci 1999;896:226-45.

6. Bonetti PO, Lerman LO, Lerman A. Endothelial dysfunction: a marker of atherosclerotic risk. Arterioscler Thromb Vasc Biol 2003;23:168-75.

7. Ponthieux A, Herbeth B, Droesch S, Haddy N, Lambert D, Visvikis S. Biological determinants of serum ICAM-1, E-selectin, P-selectin and L-selectin levels in healthy subjects: the Stanislas study. Atherosclerosis 2004;172:299-308.

8. Glowinska B, Urban M, Peczynska J, Florys B. Soluble adhesion molecules (sICAM-1, sVCAM-1) and selectins (sE selectin, sP selectin, sL selectin) levels in children and adolescents with obesity, hypertension, and diabetes. Metabolism 2005;54:1020-6.

9. Guray U, Erbay AR, Guray Y, Yilmaz MB, BoyacI AA, Sasmaz H, Korkmaz S, Kutuk E. Levels of soluble adhesion molecules in various clinical presentations of coronary atherosclerosis. Int J Cardiol 2004;96:235-40.

10. Kubzansky LD, Davidson KW, Rozanski A. The clinical impact of negative psychological states: expanding the spectrum of risk for coronary artery disease. Psychosom Med 2005;67:S10-4.

11. Steptoe A, Owen N, Kunz-Ebrecht SR, Brydon L. Loneliness and neuroendocrine, cardiovascular, and inflammatory stress responses in middle-aged men and women. Psychoneuroendocrinology 2004;29:593-611.

12. Steptoe A, Wardle J, Marmot M. Positive affect and health-related neuroendocrine, cardiovascular, and inflammatory processes. Proc Natl Acad Sci U S A 2005;102:6508-12.

13. O’Donnell K, Badrick E, Kumari M, Steptoe A. Psychological coping styles and cortisol over the day in healthy older adults. Psychoneuroendocrinology 2008;33:601-11.

14. Teper E, O'Brien JT. Vascular factors and depression. Int J Geriatr Psychiatry 2008;23:993-1000.

15. Mangos GJ, Walker BR, Kelly JJ, Lawson JA, Webb DJ, Whitworth JA. Cortisol inhibits cholinergic vasodilatation in the human forearm. Am J Hypertens 2000;13:1155-60.

16. Tsigos C, Chrousos GP. Hypothalamic-pituitary-adrenal axis, neuroendocrine factors and stress. J Psychosom Res 2002;53:865-71.

17. Whitworth JA, Mangos GJ, Kelly JJ. Cushing, cortisol, and cardiovascular disease. Hypertension 2000;36:912-6.

18. Steptoe A, Kunz-Ebrecht SR, Owen N. Lack of association between depressive symptoms and markers of immune and vascular inflammation in middle-aged men and women. Psychol Med 2003;33:667-74.

19. Pollitt RA, Daniel M, Kaufman JS, Lynch JW, Salonen JT, Kaplan GA. Mediation and modification of the association between hopelessness, hostility, and progression of carotid atherosclerosis. J Behav Med 2005;28:53-64.

20. Kiecolt-Glaser JK, Preacher KJ, MacCallum RC, Atkinson C, Malarkey WB, Glaser R. Chronic stress and age-related increases in the proinflammatory cytokine IL-6. Proc Natl Acad Sci U S A 2003;100:9090-5.

21. Appels A, Bar FW, Bar J, Bruggeman C, de Baets M. Inflammation, depressive symptomatology, and coronary artery disease. Psychosom Med 2000;62:601-5.

22. Kiecolt-Glaser JK, McGuire L, Robles TF, Glaser R. Psychoneuroimmunology and psychosomatic medicine: back to the future. Psychosom Med 2002;64:15-28.

23. Ridker PM, Hennekens CH, Roitman-Johnson B, Stampfer MJ, Allen J. Plasma concentration of soluble intercellular adhesion molecule 1 and risks of future myocardial infarction in apparently healthy men. Lancet 1998;351:88-92.

24. Irwin MR, Miller AH. Depressive disorders and immunity: 20 years of progress and discovery. Brain Behav Immun 2007;21:374-83.

25. Janszky I, Lekander M, Blom M, Georgiades A, Ahnve S. Self-rated health and vital exhaustion, but not depression, is related to inflammation in women with coronary heart disease. Brain Behav Immun 2005;19:555-63.

26. Whooley MA, Caska CM, Hendrickson BE, Rourke MA, Ho J, Ali S. Depression and inflammation in patients with coronary heart disease: findings from the heart and soul study. Biol Psychiatry 2007;62:314-20.

27. Rajagopalan S, Brook R, Rubenfire M, Pitt E, Young E, Pitt B. Abnormal brachial artery flow-mediated vasodilation in young adults with major depression. Am J Cardiol 2001;88:196-8.

28. Morenoff JD, House JS, Hansen BB, Williams DR, Kaplan GA, Hunte HE. Understanding social disparities in hypertension prevalence, awareness, treatment, and control: the role of neighborhood context. Soc Sci Med 2007;65:1853-66.

29. Whipple MO, Lewis TT, Sutton-Tyrrell K, Matthews KA, BarinasMitchell E, Powell LH, Everson-Rose SA. Hopelessness, depressive symptoms and carotid atherosclerosis in women: the Study of Women's Health Across the Nation (SWAN) heart study. Stroke 2009;40:3166-72.

30. Kohout FJ, Berkman LF, Evans DA, Cornoni-Huntley J. Two shorter forms of the CES-D Depression Symptoms Index. J Aging Health 1993;5:179-93.

31. Radloff L. A self-report depression scale for research in the general population. App Psych Measurement 1977;1:385-401.

32. Pandya R, Metz L, Patten SB. Predictive value of the CES-D in detecting depression among candidates for disease-modifying multiple sclerosis treatment. Psychosomatics 2005;46:131-4.

33. Blake GJ, Ridker PM. Novel clinical markers of vascular wall inflammation. Circ Res 2001;89:763-71.

34. Blann AD, Lip GYH. Hypothesis: is soluble P-selectin a new marker of platelet activation? Atherosclerosis 1997;128:135-8.

35. Linke SE, Rutledge T, Johnson BD, Vaccarino V, Bittner V, Cornell CE, Eteiba W, Sheps DS, Krantz DS, Parashar S, Bairey Merz CN. Depressive symptom dimensions and cardiovascular prognosis among women with suspected myocardial ischemia: a report from the National Heart, Lung, and Blood Institute-sponsored women's ischemia syndrome evaluation. Arch Gen Psychiatry 2009;66:499-507.

36. Mosesson M, Kevin R. Siebenlist David A. Meh. The structure and biological features of fibrinogen and fibrin. Ann N Y Acad Sci 2001; 936:11-30.

37. Blankenberg S, Barbaux S, Tiret L. Adhesion molecules and atherosclerosis. Atherosclerosis 2003;170:191-203.

38. Anda R, Williamson D, Jones D, MacEra C, Eaker E, Glassman A, Marks J. Depressed affect, hopelessness, and the risk of ischemic heart disease in a cohort of U.S. adults. Epidemiology 1993;4:285-94. 